\title{
People's V-Formation and Side-by-Side Model Adapted to Accompany Groups of People by Social Robots
}

\author{
Ely Repiso $^{\dagger} \quad$ Francesco Zanlungo $^{\ddagger} \quad$ Takayuki Kanda $^{\ddagger}, \S \quad$ Anaís Garrell $^{\dagger} \quad$ Alberto Sanfeliu $^{\dagger}$
}

\begin{abstract}
This paper presents a new method to allow robots to accompany a person or a group of people imitating pedestrians behavior. Two-people groups usually walk in a side-by-side formation and three-people groups walk in a Vformation so that they can see each other. For this reason, the proposed method combines a Side-by-side and V-formation pedestrian model with the Anticipative Kinodynamic Planner (AKP). Combining these methods, the robot is able to do an anticipatory accompaniment of groups of humans, as well as to avoid static and dynamic obstacles in advance, while keeping the prescribed formations. The proposed framework allows also a dynamical re-positioning of the robot, if the physical position of the partners change in the group formation. Furthermore, people have a randomness factor that the robot has to manage, for that reason, the system was adapted to deal with changes in people's velocity, orientation and occlusions. Finally, the method has been validated using synthetic experiments and real-life experiments with our Tibi robot. In addition, a user study has been realized to reveal the social acceptability of the method.
\end{abstract}

\section{INTRODUCTION}

In some years, robots are going to share environments with humans, for this reason, their behavior should be accepted by people, therefore, robots will need to understand human behaviors and to reproduce them, while doing some tasks with them. For instance, the well-known "companion robot", which is a robot moving in a human crowd accompanying one or more pedestrians (e.g. for assisting them in some task [1], guiding them [2]-[4], for recreational purposes or even as an autonomous vehicle -e.g. a wheelchair- [5]) would clearly also need to be able to move as part of a group.

Specific to navigation tasks in urban environments, walking formation is one of the most important problems. In these environments, people tend to walk in groups. Humans are

Work supported by the Spanish Ministry of Science and Innovation under project ColRobTransp (DPI2016-78957-RAEI/FEDER EU), RoboCom++ (FLAG-ERA JCT 2016), by the Spanish State Research Agency through the María de Maeztu Seal of Excellence to IRI (MDM-2016-0656), and by JST CREST (JPMJCR17A2). Ely Repiso is also supported by Spanish Ministry of Science and Innovation under a FPI-grant, BES-2014-067713. This stay was supported by Spanish Ministry of Science and Innovation under an Aid to predoctoral mobility for short stays in R D centers 2017, EEBB-I-18-12958.

${ }^{\dagger}$ The authors are with the Institut de Robòtica i Informàtica Industrial (CSIC-UPC). Llorens Artigas 4-6, 08028 Barcelona, Spain. \{erepiso, agarrell, sanfeliu\}@iri.upc.edu

$¥$ The authors are associated with ATR Intelligent Robotics Laboratories, 2-2-2 Hikaridai Seika-cho Soraku-gun, Kyoto, 619-0288, Japan. \{zanlungo, kanda\}@atr.jp

$\S$ The author is associated with Kyoto University, Yoshida-Honmachi Sakyo-ku, Kyoto, 606-8501, Japan. kanda@i.kyoto-u.ac.jp
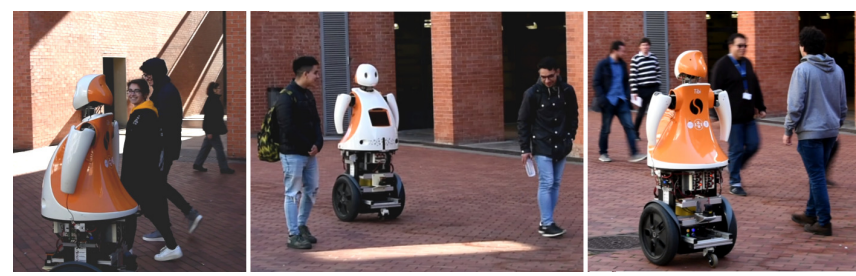

Fig. 1. Real-life experiments in the Barcelona Robot Lab. Left: Tibi accompanies the volunteers in one side of the V-formation. Center: Tibi accompanies the volunteers in the middle of the Vformation. Right: Tibi accompanies one volunteer in Side-by-side formation

able to naturally walk in different formations, depending on the number and type of group members, while navigating the environment. Two people groups typically walk in a side-by-side formation; three people groups usually walk in a V-formation [6]. Then, in order to make robots capable of navigating together with people, it is very important that robots have the capacity to join side-by-side formation and V-formation, Fig. 1. While obviously also larger groups with 4 or more members are present in pedestrian crowds, 2 and 3 people groups are more frequent and are the only ones with a stable spatial structure, while larger groups tend to split in two and three people subgroups [7]-[12]. Furthermore, due to occlusion, it may be very difficult to track all the members of a large group using the robot's sensors. Then, it appears thus natural to focus for the moment on an explicit implementation limited to groups of 2 or 3 components.

Walking in groups is not at all trivial for robots, because this involves complex computations. Robots have to perform several complex tasks at the same time, such as: to infer the final destination and the best path to go through; to take into account the orientation of the movement of the group; to adapt their desired velocity to the changes of the people's velocity (accelerating, decelerating and even stopping when necessary); to maintain the formation and to be able to change their position in the group if people change their positions; to always detect their companions or at least include a behavior to deal with people's occlusions by other members of the group; and, finally, to anticipate the behavior of all pedestrians to avoid collisions in advance. In this work, all these problems have been addressed to achieve the goal of accompanying groups of people.

In the remainder of the paper, the related work is presented in Sec. II. Sec. III exposes the implemented approach. The metrics of performances are introduced in Sec. IV. 
Sec. V describes the results of synthetic experiments and real-life experiments. Sec. VI present an user study. Finally, conclusions are given in Sec. VII.

\section{RELATED WORK}

This section presents the related work on models of robot companions. Several approaches to accompany and follow people with several robots have been developed [13]-[15], but very few of these consider more than one person being accompanied by the robot [16]-[18]. Furthermore, these works tend to see the robot as a guide, not as a companion or coworker that is part of the group.

Authors in [14] proposed a model of people walking sideby-side which could predict the partners future position, and subsequently generate a plan. In Repiso et al. [19] the authors proposed a method for on-line adaptive side-by-side for human robot companion in dynamic urban environments and in [20] the authors extended the method to also approach a moving person to interact. In [21], researchers implemented a robot that follows people to obtain a more realistic and human-like navigation in crowded scenarios. Most of the companion approaches with only one person try to adapt the robot's behavior to that of the people being accompanied, e.g. by trying to infer the behavior of the latter [15].

Regarding the approaches that take into account more than one person and more than one robot, we can find works as, Saez et al. [17] that develop a system to allow a group of robots to move coordinately with a human. Urcola et al. [18] presented a guiding behavior for a team of robots, where the leader of the formation was the guiding robot and the other robots were the followers that were situated behind the group of people. A previous work [22] implements a guiding procedure for a group of robots, by using a leader robot that guides people and several shepherding robots that try to regroup people and maintain the cohesion of the group.

While other studies only take into account one person involved in the task or only maintain the cohesion of a group of people to guide them, we implemented a method that uses a pedestrian model to obtain a better accompaniment of a group of people. Also, our robot tries to adapt to the people behaviour in terms of velocity and walking direction, while maintains the formation with them. Furthermore, we propose a solution for the common problem of occlusion of people inside one group to be able of accompany them taking into account all members of the group.

\section{METHOD}

This section explains the method implemented to allow a robot to accompany a group of people. First, the navigation framework is presented in Section III-A. Second, the new method to accompany a group of two people in a $\mathrm{V}$-formation is explained in Section III-B (for the implementation of Side-by-side walking, refer to the 2-people group description in [6]). Furthermore, as discussed in [6], this model may be used also for larger groups, although for the reasons explained in the introduction we limit ourselves to a two and three group member implementation.

\section{A. Human-Robot Companion Navigation Framework.}

Groups of people can have several destinations in any environment. Due to that, robots accompanying humans have to infer from people's movement the most suitable destination. In this work, we make use of the Human Motion Intentionality Predictor (BHMIP) [23] to select the most probable final destination of the group from all possible destinations in the environment. These destinations were predefined in locations such as: work places, entrances and exits of the environment. Also, the method predicts the movement of all tracked pedestrians of the environment, including members of the group, during a window of time of 5 seconds to anticipate their behavior, by using the BHMIP and the Social Force Model (SFM) [16].

Once the final destination of the group is obtained, all possible paths are computed by the Anticipative Kinodynamic Planner (AKP) [24], and extended to accompany people [20]. This extension includes a force for the accompaniment task and a cost to evaluate the accompaniment task during each path.

The AKP computes all the paths using the SFM, with the following equation:

$$
\mathbf{F}_{r}=\alpha \mathbf{f}_{r, d}^{\text {goal }}+\beta \mathbf{F}_{r, p}^{\text {group }}+\left(\gamma \mathbf{F}_{r}^{\text {ped }}+\delta \mathbf{F}_{r}^{\text {obs }}\right)
$$

$\alpha, \beta, \gamma$ and $\delta$ were obtained as described in [13].

The force towards the group destination, that assumes that the robot adapts its velocity to reach the predicted common goal of the group, is defined in Eq. 2.

$$
\left.\mathbf{f}_{r, d}^{\text {goal }}=k\left(\mathbf{v}_{r}^{0}\left(d_{n}{ }^{g}\right)-\mathbf{v}_{r}\right)\right)
$$

where $\mathbf{v}_{r}$ and $\mathbf{v}_{r}^{0}\left(d_{n}{ }^{g}\right)$ are, respectively, the current and desired (e.g., directed towards the goal and with a suitable speed) velocities of the robot. The therms, $\mathbf{F}_{r}^{\text {ped }}$ and $\mathbf{F}_{r}^{\text {obs }}$, are two repulsive forces to avoid pedestrians and obstacles outside the group, see [13].

Finally, Eq. 3 shows the force used by the robot to accompany people when moving in a group.

$$
\mathbf{F}_{r, p}^{\text {group }}=\sum_{j \in P_{c}} \mathbf{f}_{r, j}^{\text {group }},
$$

where $r$ means robot and $p$ means pedestrians and $P_{c}$ is the set of all people that the robot accompanies. The force $\mathbf{f}_{r, j}^{\text {group }}$ can be $\mathbf{f}_{r, j}^{\text {first }}$ or $\mathbf{f}_{r, j}^{\text {second }}$ depending on the distance between person and robot. These forces are explained in next Sub-Section (Eqs. 8 and 9).

Once all possible paths that the robot may perform to accompany the group until the final destination are computed, the optimal one needs to be selected. The evaluation is done through the use of a cost function that considers several characteristics of the paths:

$$
\mathbf{J}\left(S, s_{\text {goal }}, U\right)=\left[J_{d}, J_{o r}, J_{r}, J_{p}, J_{o}, J_{c}\right]
$$

The terms in the equation, from left to right, evaluate the following characteristics: the distance to reach the goal, the difference between the real orientation of the robot and the desired orientation to reach the goal, the difficulty of 
the robot control to do the path, the work of the robot to avoid pedestrians and obstacles, and the performance of the companion task. For more information about the costs that evaluate the best path, the reader is referred to [19], [25]. Also, we include a brief explanation of the group behavior cost function in Sub-Section III-B.

After computing each individual cost function, the method uses two more calculations to obtain a single-objective cost function. First, to avoid the scaling effect of weighted-sum method, each cost function is normalized to $(-1,1)$, using:

$$
\bar{J}_{i}(\mathbf{X})=\operatorname{erf}\left(\frac{x-\mu_{x}}{\sigma_{x}}\right)
$$

The mean, $\mu_{x}$, and variance, $\sigma_{x}$, will be estimated after all paths are computed.

Finally, a weighted sum $J: R^{I} \rightarrow R$ is computed to obtain the final cost, Eq. 6; for additional explanation the reader is pointed to [25].

$$
J\left(S, s_{\text {goal }}, U\right)=\sum_{i} w_{i} \bar{J}_{i}\left(S, s_{\text {goal }}, U\right) .
$$

\section{B. Three people group model (V-formation)}

Here, we proceed to describe the 5-steps method to achieve the three people group V-formation.

1) Model formulation: In [6], the authors introduced a mathematical model, based on a pair-wise interaction potential, describing the dynamics of socially interacting pedestrian groups. Writing the relative position between two socially interacting pedestrians $i$ and $j$ as $\mathbf{r}_{i j} \equiv \mathbf{r}_{i}-\mathbf{r}_{j}=$ $\left(r_{i j}, \theta_{i j}\right)$, where $\theta=0$ gives the direction to the pedestrians' goal, the discomfort of $i$ due to not being located in the optimal position for social interaction with $j$ is given by the (discomfort) potential ${ }^{1}$

$$
\begin{gathered}
U^{\eta}\left(r_{i j}, \theta_{i j}\right)=R\left(r_{i j}\right)+\Theta^{\eta}\left(\theta_{i j}\right), \\
R(r)=C_{r}\left(\frac{r}{r_{0}}+\frac{r_{0}}{r}\right), \\
\Theta^{\eta}(\theta)=C_{\theta}\left((1+\eta) \theta^{2}+(1-\eta)(\theta-\operatorname{sign}(\theta) \pi)^{2}\right),
\end{gathered}
$$

where $r_{0}$ is the most comfortable interaction distance, and $-1 \leq \eta<0$ is related to the intensity of social interaction. The acceleration of the pedestrian $i$ due to group dynamics, i.e. the action of the pedestrian aimed to minimize social interaction discomfort with respect to $j$, is given by

$$
\mathbf{f}_{i j}^{f i r s t}=-\nabla_{i} U^{\eta}\left(\mathbf{r}_{i j}\right)
$$

In the potential of Eq. 7, the radial term $R$ assures that the pedestrians will have a distance close to $r_{0}$, while the angular potential $\Theta^{\eta}$ allows them to keep both their interaction partner and their walking goal in sight (the more negative $\eta$ is, the more pedestrians will try to have interaction partners in their vision field). In a pedestrian group, first neighbors interact through the force of Eq. 8, while second neighbors

\footnotetext{
${ }^{1}$ In Eq. 7, we are assuming $-\pi<\theta<\pi$, and using $\operatorname{sign}(0)=-1$ in order to have a continuous potential. Refer to the original work for details.
}

interact through a weaker and simplified potential depending only on the radial potential of Eq. 9, [26].

$$
\mathbf{f}_{i j}^{\text {second }}=-\frac{1}{2} \nabla_{i} R\left(r_{i j}\right),
$$

In this model, a pedestrian's neighborhood is decided based on its position on the axis orthogonal to the direction of the goal, see [26] for details.

The model, in agreement with observational data, predicts that 3 people group:

- are slower than individual pedestrians, and in particular the velocity of the group is given by

$$
v^{\text {group }} \approx v^{p}+\frac{8 C_{\theta} \eta \pi}{3 r_{0} \kappa}<v^{p}
$$

where $v^{p}$ is the individual preferred velocity for people, the analog velocity for the robot is represented by the desired velocity of the robot, $\mathbf{v}_{r}^{0}\left(d_{n}{ }^{g}\right)$, in Eq. 2 .

- walk in a V-formation, with the central pedestrian walking slightly behind. This formation is believed to facilitate communication between all members of the group.

Furthermore, when combined with proper path planning and collision avoidance, the model provides a very effective and natural preservation of group structure while navigating in complex environments [27].

We would like to stress that this mathematical model is not a prescription to walk in a $\mathrm{V}$-formation in a 3 pedestrian setting, but a more general $\mathrm{N}$-pedestrian model that reproduces 2 people Side-by-side and 3 people V-formations in absence of further constraints (e.g. collision avoidance). While in this section we provided details related to the more complex 3 people groups, we also used this model for the implementation of Side-by-side walking.

2) Robot preferred velocity: The model described above assumes that pedestrians moving in a group have (or agree on) a similar preferred velocity. Due to Eq. 10, nevertheless, this is not the velocity at which the group moves. Since the proposed system is based on a Social Force Model framework, the robot will need to be provided with the correct preferred velocity, which is, as stated above, different from the observed velocity of the companions. This is not, nevertheless, a serious limitation, at least from a theoretical viewpoint. We may indeed define the velocity of the group $v^{\text {group }}$ as the average of the observed velocities of the companions, compute the preferred velocity $v^{p}$ by inverting Eq. 10, and pass the result to the robot as its SFM preferred velocity.

3) Cost function: By representing the "discomfort" of the pedestrian for not being in an optimal position with respect to the companions, the potential of Eq. 7 is the most natural candidate as a cost function for the "companion behavior" when using the model of [6] in the proposed robotcompanion system. Similarly, the potential may be used to define a performance metric that assumes values in $[0,1]$, as explained in the next section. 
4) Deal with occlusion problems: In the V-formation model there are two possible types of formations. One with the robot in the middle of the formation, and other with the robot in one of the sides. When the robot is on one side, the system has to deal with partial or total persons' occlusions. Then, to deal with these situations, the system generates a fictitious person behind the human that the robot can detect, aligned in the direction of occlusion of the laser of the robot.

5) Deal with direction changes until the goal: The method includes a final goal modification. That is, the robot modifies the position of the predefined destinations in the environment to adapt to people movement. When the robot computes the final destination of the group, it takes into account its current position and the group's orientation:

$$
\begin{gathered}
m=\tan \frac{v_{y}^{\text {group }}}{v_{x} \text { group }} \\
\left\{\begin{array} { l } 
{ y _ { r } = m x _ { r } + b _ { 1 } } \\
{ y _ { d } = - \frac { 1 } { m } x _ { d } + b _ { 2 } }
\end{array} \left\{\begin{array}{l}
x_{n d}=\frac{b_{2}-b_{1}}{m+\frac{1}{m}} \\
y_{n d}=m x_{n d}+b_{1}
\end{array}\right.\right.
\end{gathered}
$$

Here, $\left(v_{x}^{\text {group }}, v_{y}^{\text {group }}\right)$ is the average of the observed velocities of the people that accompanies the robot, $\left(x_{r}, y_{r}\right)$ is the robot's position, $b_{1}$ and $b_{2}$ are calculated using the previous equations, and $\left(x_{n d}, y_{n d}\right)$ is the new final destination of the robot. The new destination, $\left(x_{n d}, y_{n d}\right)$, allows the robot to move with the group in different directions, that may differ from the final goal. This functionality is useful if there are few destinations or far destinations, or there exist several obstacles until the final goal.

\section{PERformance Metrics}

To define a performance metric that assumes values in $[0,1]$ ( 0 representing the minimum value, i.e. inability to keep the $\mathrm{V}$-formation, and 1 the maximum value, i.e. moving in a perfect V-formation), we proceed in the following way. We define a "maximum allowable potential value" $U_{\max }$ for the system, which corresponds to a formation in which all the pedestrians and robot walk abreast (acceptable formation), but the distance between the robot and one of the partners is $\bar{r}=0.5$ meters (that we define as the minimum acceptable distance to avoid collisions), while the distance to the other pedestrian is $r_{0}$. Then we can calculate $U_{\max }$ by substituting these values in Eqs. 7, 9 obtaining Eq. 13 for the robot in the lateral and Eq. 14 for the robot in the center of the group. For a robot walking on the side of the group this is

$$
U_{\max }^{\text {side }}=C_{r}\left(1+\frac{\bar{r}}{r_{0}}+\frac{r_{0}}{\bar{r}}\right)+C_{\theta} \frac{\pi^{2}}{2}
$$

while for a robot in the central position it is

$$
U_{\text {max }}^{\text {center }}=C_{r}\left(2+\frac{\bar{r}}{r_{0}}+\frac{r_{0}}{\bar{r}}\right)+C_{\theta} \pi^{2}
$$

We can then numerically compute (e.g., by evaluating Eqs. 7, 9 in each point of a grid) the minimum value allowed for the potential ${ }^{2}$ of the robot given the position of the two

\footnotetext{
${ }^{2}$ In the potentials a negative velocity dependent linear term in the direction of motion has to be added to account for the drift term. The arbitrary constant has been chosen so that the terms disappear in Eqs. 13, 14.
}

companions, $U_{m i n}$, and the current potential of the robot $U_{\text {current }}$ (Eqs. 7, 9), and define the metric $M$ as

$$
M= \begin{cases}0, & \text { if } U_{\text {current }}>U_{\max } \\ \frac{U_{\max }-U_{\text {current }}}{U_{\max }-U_{\min }}, & \text { otherwise }\end{cases}
$$

We have $M \approx 0$ every time the robot gets dangerously close to one of the pedestrians, and in any other configuration that it is held similarly "not comfortable" for pedestrian interaction by the model of [6], and $M \approx 1$ when the robot accomplishes to walk in a V-formation similar to the one observed in human pedestrians. Nevertheless, it has to be stressed that the "perfect V-formation" ( $M \approx 1)$ is observed only for pedestrians that walk in wide environments with low density crowd, and thus the metric defined above is expected to assume lower values in presence of many obstacles and pedestrians external to the group. The metric for the Sideby-side case is defined in an equivalent way, i.e. by using in Eq. 13 the two people potential of Eq. 7 to describe the worst acceptable behavior.

\section{EXPERIMENTS}

This section addresses the synthetic and real-life experiment results. First, the proposed method was tested in simulation using several environments and reproducing different real-life situations. The method was then tested in real-life experiments, using the robot Tibi of the Institut de Robotica i Informatica Industrial (IRI). This robot has a maximum speed of $1 \mathrm{~m} / \mathrm{s}$, due to safety reasons. Accordingly, the robot is able to accompany and adapt its behavior to people who walk at speeds below $0.8 \mathrm{~m} / \mathrm{s}$.

\section{A. Synthetic Experiments}

The simulation environment is composed of several elements. Firstly, the robot follows the method to accompany people using the proposed group model behavior in combination with the AKP navigation model (the Anticipative Kinodynamic Planner). As we have commented, the robot is non-holonomic and has a maximum velocity of $1 \mathrm{~m} / \mathrm{s}$. The robot is represented in the simulated environment by the Tibi's model. Secondly, one or two accompanied people are simulated and walk according to AKP, which gives more realistic accompany behavior. The predictions of the people accompanied by the robot are represented in blue, when they are detected by the robot; and in red when the second person is not detected by the robot. Also, all people are represented as green cylinders with identification numbers over them. Thirdly, other simulated pedestrians walk randomly toward different destinations in the environment. These people use also the Extended Social Force Model (ESFM) to avoid static obstacles, other people and the robot. We have several people with a reactive behavior. All people walk randomly with velocities in the interval $[0-0.8] \mathrm{m} / \mathrm{s}$. Finally, the environment includes several static obstacles positioned in different places. In the images of the simulated environment these obstacles are represented by gray surfaces. Images of the simulated environments in Fig. 2 and 3. 


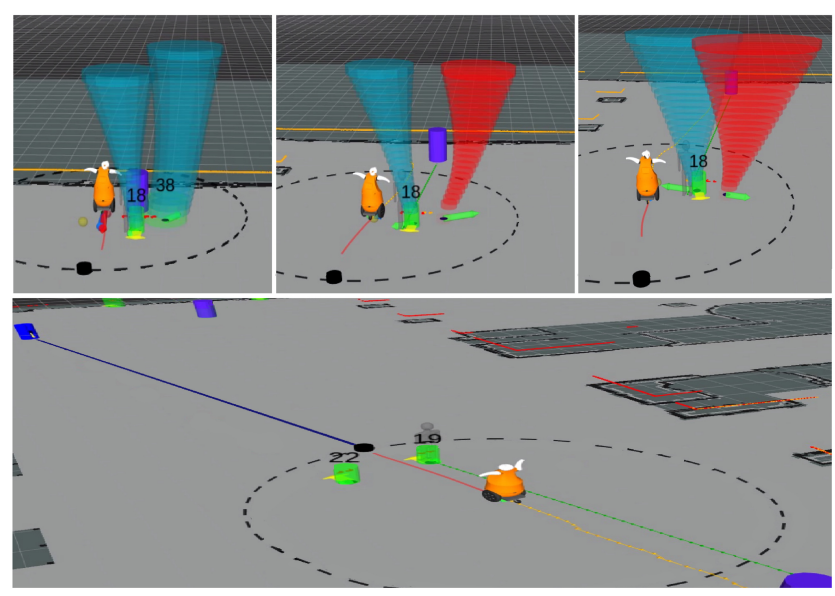

Fig. 2. Synthetic experiments: Simulation environment to test and evaluate the new method to accompany groups of people. $U p$ : Computation of the fictitious person to deal with people occlusions inside the group. Down: Dynamic final goal to include the orientation of the people of the group to go until the final destination. The purple cylinders are the static final destinations of the environment.

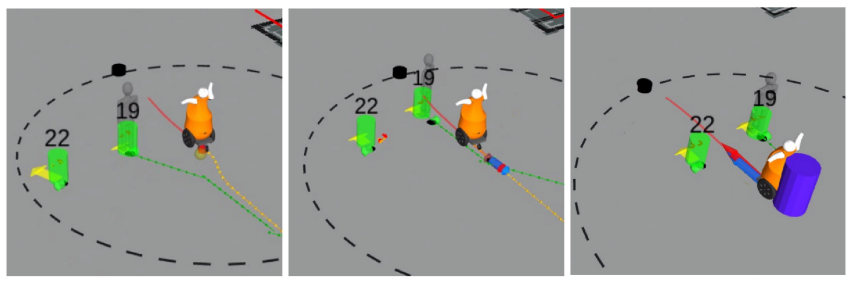

Fig. 3. Synthetic experiments: Adaptation of the robot's physical position when the partners change its physical position in the group formation.

Moreover, the simulated environment has other elements, such as the dynamic final destination represented by a darkblue cylinder, the window of the local planner shown as a black dashed circle around the robot, the local plan that is drawn in red and the global plan that is drawn in dark blue.

More than 1.900 simulations were performed to test and validate the new model. The simulation environment reproduces several real-life situations, which are described below. Firstly, the environment allowed to test the method without any type of obstacles to see the appropriate group behavior of the robot. When the robot accompanies one person, it does so by positioning itself on one of the sides of the person (performance of side-by-side Table. I). Then, when the robot accompanies a group of two people, it does so by positioning itself from three different physical positions (right, center, left) to reach different destinations (performance of V-formation Table. I). These destinations were used to test the method for different orientations of the group, within an environment. To obtain a better accompaniment performance, the robot used the orientation of the movement of the group to recalculate each time the best position of the final destination, Fig. 2-down. In these simulations we observed how the robot was able to adapt its position dynamically if the people rearrange their physical position inside the group, Fig. 3, and how it was able to adapt its preferred velocity to follow the people's group velocity, Fig. 4. Secondly, In the case that the robot accompanies two people, the simulation environment

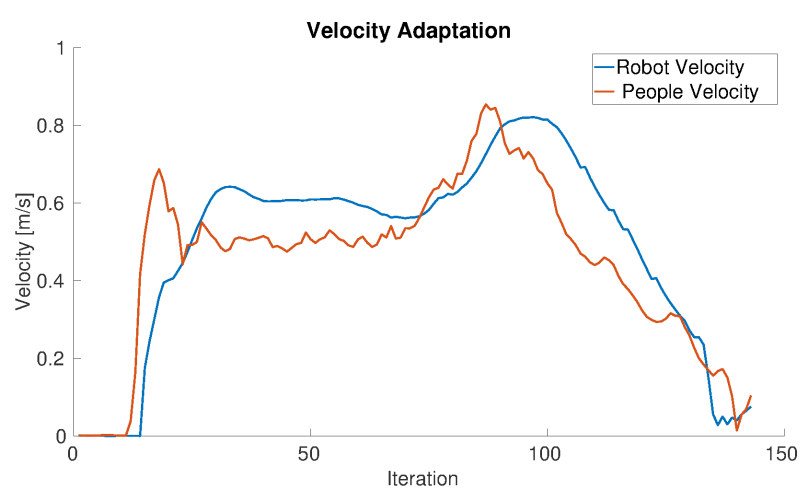

Fig. 4. Velocity adaptation: The graph shows how the robot adapts its velocity to the people velocity. This people velocity is the mean of the detected velocity of all the people of the group.

\begin{tabular}{|l|c|}
\hline & Performance \\
Side-by-side & $0.89( \pm 0.024)$ \\
Side-by-side with static and dynamic obstacles & $0.81( \pm 0.08)$ \\
V-form robot in side & $0.84( \pm 0.044)$ \\
V-form robot in middle & $0.83( \pm 0.09)$ \\
V-form with fictitious person & $0.75( \pm 0.049)$ \\
V-form with static and dynamic obstacles & $0.61( \pm 0.09)$ \\
\hline
\end{tabular}

TABLE I

The results of Performance of the accompaniment of the robot of the simulation experiments for all the simulated cases.

represented situations where one of the accompanied person occludes the other, to test the new robot behavior to deal with people occlusions, Fig. 2-up. When the robot could not detect one of the accompanied persons, it generated a fictitious one in the position where the person should be located, behind the person who was correctly detected. With the position of the fictitious person, the robot was able to maintain the $\mathrm{V}$-formation (performances of V-form with fictitious person, in Table I). Thirdly, the simulations included static obstacles and random pedestrians walking through random destinations in the environment. These experiments allowed to study how the robot anticipates the other people behavior and future collisions with obstacles. To deal with these future interactions, the robot compresses or dynamically changes the formation of the group. Furthermore, if other people blocked the path of the group until the destination, the group stopped until pedestrians pass through. If only people of the group were blocked, the robot would wait until all the group could walk again (performances of Side-by-side and V-form with static and dynamic obstacles in Table. I).

The reader can see all the results in terms of performance in Table. I. The results are expressed in a scale between 0 and 1 , where 1 is the best value of performance and the value between brackets is the standard error of each mean value. These performances were calculated with the performance metric of Eq. 15.

\section{B. Real-life Experiments}

The real-life experiments were done with the Tibi robot in the Facultat the Matematiques and Estatistics (FME) and in the Barcelona Robot Lab (BRL), situated in the Campus nord 


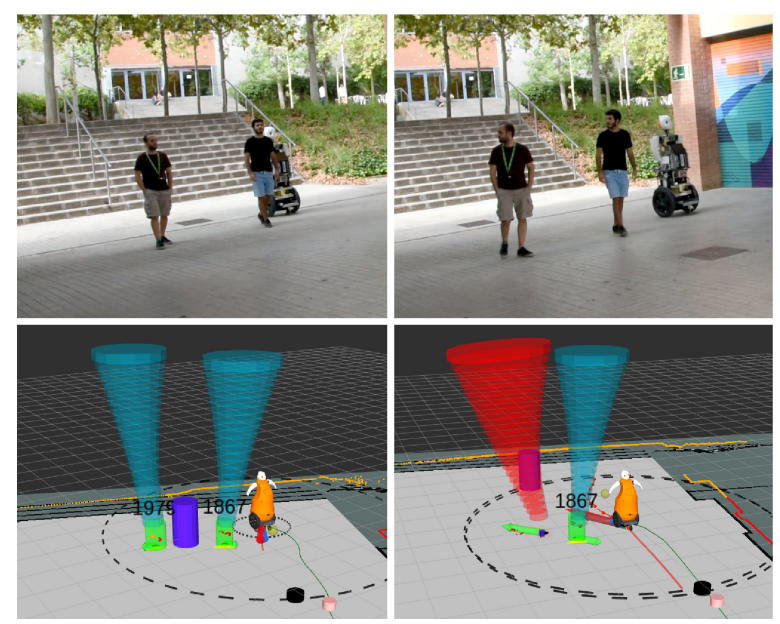

Fig. 5. Real-life experiments in the FME: Tibi generates the fictitious person, because one person is occluded by the other.

of the Universitat Politecnica de Catalunya (UPC). This robot is designed to operate in urban pedestrian areas. The robot is socially accepted and humans take interest in interacting with it. All people in the environment were detected using a laser leg detector and tracked using a multi-hypothesis tracker [28] that differentiates each people using an identifier. Then, as differentiating the accompanied people is out of the scope of this paper, we selected these people by proximity at the beginning of the experiment and keep track of them during all the experiment.

In the real-life experiments several groups of volunteers were accompanied to reach different destinations. The robot could accompany a person doing a Side-by-side formation or accompany a group of two people doing a V-formation, in which case the robot could be situated in three possible physical positions (right, center and left), Fig. 1. Fig. 5 shows a case of occlusion and the posterior creation of the fictitious person. More than 70 people participated in the experiments, in pairs or alone, and no instruction were given to the volunteers, besides telling them that Tibi accompanied them and they had to walk as they preferred. Also, they may change their positions inside the formation and the robot kept doing his job well. Table II shows the performance results for the real-life experiments. The difference between the performances in the FME and BRL is due to the space that is available in each environment, since the method has an initialization period where the robot is not yet in the desired position. Notice that the results are expressed in a scale between 0 and 1 . The value between brackets is the standard error of each mean value. These performances were calculated with the performance metric of Eq. 15.

We would like to address the reader to see the videos of the real-life experiments in the following link http://www . iri.upc.edu/people/erepiso/IROs2019.html.

\section{USER STUDY}

The results presented previously demonstrate that the robot is able to accompany people doing different formations depending on the number of people that the robot accompanies. Furthermore, a user study was conducted to determine

\begin{tabular}{|l|c|c|}
\hline & \multicolumn{2}{|c|}{ Performance of Accompaniment } \\
\cline { 2 - 3 } Side-by-side & Real on FME & Real on BRL \\
V-form robot in side & $0.74( \pm 0.089)$ & $0.86( \pm 0.03)$ \\
V-form robot in middle & $0.63( \pm 0.083)$ & $0.78( \pm 0.043)$ \\
\hline
\end{tabular}

TABLE II

Performance results of the real-life experiments for all the possible formations. These results include cases without other people and with other people, as dynamic obstacles for the robot.
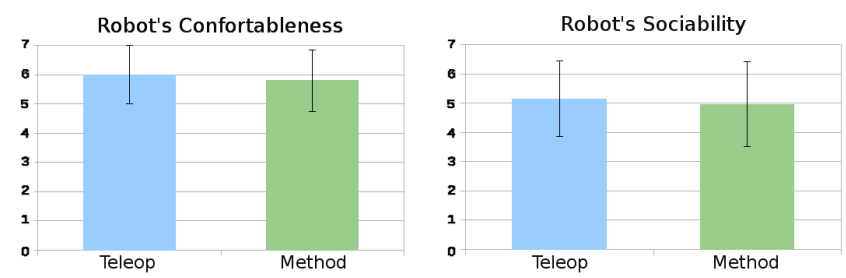

Fig. 6. User study results. Left: Robot's Comfortableness felt by the volunteers. Right: Robot's Sociability felt by the volunteers.

whether if the use of the model enhances the companion behavior of the robot, by comparing the performance of the proposed method against teleoperation by an expert, and we should highlight that people perceived that the proposed method performs similarly to the teleoperation approach.

In the experiments, Tibi was accompanying the participants in one of three possible formations (side-by-side, central V-form and lateral V-form) walking between different locations of the environment. Furthermore, other pedestrians were walking around the campus or watching the experiment and some times interfered in the way of the robot reproducing situations of obstacle avoidance's during the companion behavior. 174 real-life experiments were performed in the FME and in North Campus with Tibi: 87 using the method and 87 controlling the robot by teleoperation. The teleoperator used the following rules: try to imitate the Side-by-side or V-formation behavior of people walking in groups, while the robot walk with them in any of the positions. The participant age range was between [11-58] years old. $70.8 \%$ were men and $29.2 \%$ were women. In the study, we included a questionnaire concerning the level of knowledge of the participant in the field of robotics. We used a 7-point scale from "Not at all" to "Very much" knowledge in the field. The results in terms of average and standard deviation were $\mathrm{M}=3.03$ and $\mathrm{SD}=2.77$, which allows us to conclude that large part of the participants were potential users not related with robotics.

Social Scales: Participants were asked a set of questions following about the experience during the accompaniment task. All questions were asked on a 7-point scale from "Not at all" to "Very much". To analyze their responses, we grouped the survey questions into two scales: the first measured the robot's sociability, while the second evaluated robot's comfortableness felt by the volunteers. Both scales surpassed the commonly used 0.71 level of reliability, Cronbach's alpha. Each scale response was computed by averaging the results of the survey questions comprising 
the scale. ANOVAs were run on each scale to highlight differences between the two robot's behaviors, teleoperation and method. For the robot's sociability and Comfortableness score plotted in Fig. 6, pairwise comparison with Bonferroni demonstrate no statistical difference between the two kind of navigation approaches, $p>0.5$.

\section{CONCLUSIONS}

This paper presented a new method to accompany people in groups of two or three members using a mobile autonomous robot. The proposed algorithm combines the Side-by-side and V-formation pedestrian model [27], with the Anticipative Kinodynamic Planner (AKP) [29].

The major contributions of this paper are three-fold: First, we obtained dynamical behavior for the robot to accompany one or two people in a group of two or three members. Using the proposed method, the robot is able to find the best path to accompany the group, while taking into account future interactions with other people and obstacles; furthermore, the method allows the robot to maintain the Side-by-side or Vformation and dynamically change its physical position in the formation of the group if it is necessary. Second, we propose different solutions to deal with the randomness of people, as the velocity adaptation; and the dynamic goal calculation to take into account the direction of the movement of the accompanied people. Furthermore, the method is able to deal with people occlusions inside the group. Third, the method was tested in synthetic experiments (more than 1.900 simulations) and in real-life experiments (174 real-live experiments) in the FME and in the BRL environment with non-trained volunteers, obtaining good results. Furthermore, a user study showed the acceptance of the method by inexpert people, because the method was compared with a teleop performance and they found no difference between the two approaches.

\section{REFERENCES}

[1] B. Graf, J. H. Wandosell, and C. Schaeffer, "Flexible path planning for nonholonomic mobile robots," in Proc. 4th European workshop on advanced Mobile Robots, 2001, pp. 199-206.

[2] T. Kanda, M. Shiomi, Z. Miyashita, H. Ishiguro, and N. Hagita, "An affective guide robot in a shopping mall," in Proceedings of the 4th ACM/IEEE international conference on Human robot interaction, 2009, pp. 173-180.

[3] H.-M. Gross, H. Boehme, C. Schroeter, S. Müller, A. König, E. Einhorn, C. Martin, M. Merten, and A. Bley, "Toomas: interactive shopping guide robots in everyday use-final implementation and experiences from long-term field trials," in IEEE/RSJ International Conference on Intelligent Robots and Systems, 2009, pp. 2005-2012.

[4] A. Garrell, M. Villamizar, F. Moreno-Noguer, and A. Sanfeliu, "Teaching robots proactive behavior using human assistance," International Journal of Social Robotics, vol. 2, no. 9, pp. 231-249, 2017.

[5] E. Prassler, D. Bank, and B. Kluge, "Motion coordination between a human and a robotic wheelchair," in Proceedings. 10th IEEE International Workshop on Robot and Human Interactive Communication, 2001, pp. 412-417.

[6] F. Zanlungo, T. Ikeda, and T. Kanda, "Potential for the dynamics of pedestrians in a socially interacting group," Physical Review E, vol. 89, no. 1, p. 012811, 2014.

[7] M. Moussaïd, N. Perozo, S. Garnier, D. Helbing, and G. Theraulaz, "The walking behaviour of pedestrian social groups and its impact on crowd dynamics," PloS one, vol. 5, no. 4, p. e10047, 2010.

[8] A. Gorrini, S. Bandini, and M. Sarvi, "Group dynamics in pedestrian crowds: Estimating proxemic behavior," Transportation research record, vol. 2421, no. 1, pp. 51-56, 2014.
[9] M. Schultz, L. Rößger, H. Fricke, and B. Schlag, "Group dynamic behavior and psychometric profiles as substantial driver for pedestrian dynamics," in Pedestrian and Evacuation Dynamics 2012. Springer, 2014, pp. 1097-1111.

[10] W. Daamen and S. Hoogendoorn, "Controlled experiments to derive walking behaviour," European Journal of Transport and Infrastructure Research, vol. 3, no. 1, pp. 39-59, 2003.

[11] P. Zhao, L. Sun, L. Cui, W. Luo, and Y. Ding, "The walking behaviours of pedestrian social group in the corridor of subway station," in Proceedings of the 2016 Pedestrian and Evacuation Dynamics Conference, 2016.

[12] F. Zanlungo and T. Kanda, "Do walking pedestrians stabily interact inside a large group? analysis of group and sub-group spatial structure," in Proceedings of the Annual Meeting of the Cognitive Science Society, vol. 35, no. 35, 2013.

[13] G. Ferrer, A. Garrell, F. Herrero, and A. Sanfeliu, "Robot socialaware navigation framework to accompany people walking side-byside," Autonomous Robots, pp. 1-19, 2016.

[14] Y. Morales, T. Kanda, and N. Hagita, "Walking together: side by side walking model for an interacting robot," Journal of Human-Robot Interaction, vol. 3, no. 2, pp. 51-73, 2014.

[15] S. Scheggi, M. Aggravi, F. Morbidi, and D. Prattichizzo, "Cooperative human-robot haptic navigation," in Robotics and Automation (ICRA), 2014 IEEE International Conference on. IEEE, 2014, pp. 2693-2698.

[16] A. Garrell and A. Sanfeliu, "Cooperative social robots to accompany groups of people," The International Journal of Robotics Research, vol. 31, no. 13, pp. 1675-1701, 2012.

[17] J. Saez-Pons, L. Alboul, and J. Penders, "Experiments in cooperative human multi-robot navigation," in Robotics and Automation (ICRA), 2011 IEEE International Conference on. IEEE, 2011, pp. 1-4.

[18] P. Urcola and L. Montano, "Adapting robot team behavior from interaction with a group of people," in Intelligent Robots and Systems (IROS), 2011 IEEE/RSJ International Conference on. IEEE, 2011, pp. 2887-2894.

[19] E. Repiso, A. Garrell, and A. Sanfeliu, "On-line adaptive side-by-side human robot companion to approach a moving person to interact," in Iberian Robotics conference. Springer, 2017, pp. 113-125.

[20] E. Repiso, G. Ferrer, and A. Sanfeliu, "On-line adaptive side-by-side human robot companion in dynamic urban environments," in IEEE/RSJ international conference on Intelligent robots and systems. IEEE, 2017.

[21] J. Müller, C. Stachniss, K. O. Arras, and W. Burgard, "Socially inspired motion planning for mobile robots in populated environments," in Proc. of International Conference on Cognitive Systems, 2008.

[22] A. Garrell and A. Sanfeliu, "Local optimization of cooperative robot movements for guiding and regrouping people in a guiding mission," in Intelligent Robots and Systems (IROS), 2010 IEEE/RSJ International Conference on. IEEE, 2010, pp. 3294-3299.

[23] G. Ferrer and A. Sanfeliu, "Bayesian human motion intentionality prediction in urban environments," Pattern Recognition Letters, vol. 44, pp. 134-140, 2014.

[24] G. Ferrer, A. Garrell, M. Villamizar, I. Huerta, and A. Sanfeliu, "Robot interactive learning through human assistance." in Multimodal Interaction in Image and Video Applications, Book Series Intelligent Systems Reference Library, vol. 48. Springer, 2013.

[25] G. Ferrer and A. Sanfeliu, "Multi-objective cost-to-go functions on robot navigation in dynamic environments," in IEEE/RSJ international conference on Intelligent robots and systems. IEEE, 2015, pp. 38243829.

[26] F. Zanlungo and T. Kanda, "A mesoscopic model for the effect of density on pedestrian group dynamics," EPL (Europhysics Letters), vol. 111, no. 3, p. 38007, 2015.

[27] F. Zanlungo, Z. Yücel, F. Ferreri, J. Even, L. Y. M. Saiki, and T. Kanda, "Social group motion in robots," in International Conference on Social Robotics. Springer, 2017, pp. 474-484.

[28] V. Vaquero, E. Repiso, and A. Sanfeliu, "Robust and real-time detection and tracking of moving objects with minimum $2 \mathrm{~d}$ lidar information to advance autonomous cargo handling in ports," Sensors, vol. 19, no. 1, p. 107, 2019.

[29] G. Ferrer and A. Sanfeliu, "Proactive kinodynamic planning using the extended social force model and human motion prediction in urban environments," in IEEE/RSJ international conference on Intelligent robots and systems. IEEE, 2014, pp. 1730-1735. 